\title{
Monolithic optoelectronic integration of a GaAlAs laser, a field-effect transistor, and a photodiode
}

\author{
N. Bar-Chaim, K. Y. Lau, and I. Ury \\ Ortel Corporation, Alhambra, California 91803 \\ A. Yariv \\ California Institute of Technology, Pasadena, California 91125
}

(Received 9 January 1984; accepted for publication 27 February 1984)

\begin{abstract}
A low threshold buried heterostructure laser, a metal-semiconductor field-effect transistor, and a $p-i-n$ photodiode have been integrated on a semi-insulating GaAs substrate. The circuit was operated as a rudimentary optical repeater. The gain bandwidth product of the repeater was measured to be $178 \mathrm{MHz}$.
\end{abstract}

PACS numbers: 42.82. $+\mathrm{n}$, 42.55.Px, 85.60.Dw, 85.30.Tv

Recent efforts on optoelectronic integration have produced monolithic circuits incorporating lasers and transistor drivers, and photodetector and transistor amplifiers, ${ }^{1}$ but thus far integration on the same chip of all the three key components (lasers, detectors, and transistor amplifiers) has not been attained with state of the art devices. In this letter we report the first monolithic integration of a low threshold laser, a metal-semiconductor field-effect transistor MESFET, and a $p-i-n$ photodiode on a semi-insulating (SI) GaAs die whose dimensions are $325 \times 625 \mu \mathrm{m}$. By suitably interconnecting the devices, the circuit was operated as a rudimentary optical repeater, with the photodiode serving as the photodetector, the MESFET as the amplifier, and the laser as the optical emitter. This simple circuit configuration serves to illustrate the basic principles of operation of integrated optoelectronic circuits (IOEC) in general, and sheds light on the expected performance characteristics and suitable designs of more complicated repeater circuits.

A schematic diagram of the IOEC unit is shown in Fig. 1. Light incident on the photodiode causes a current to flow through the load resistor $R_{d}$. The change in current which

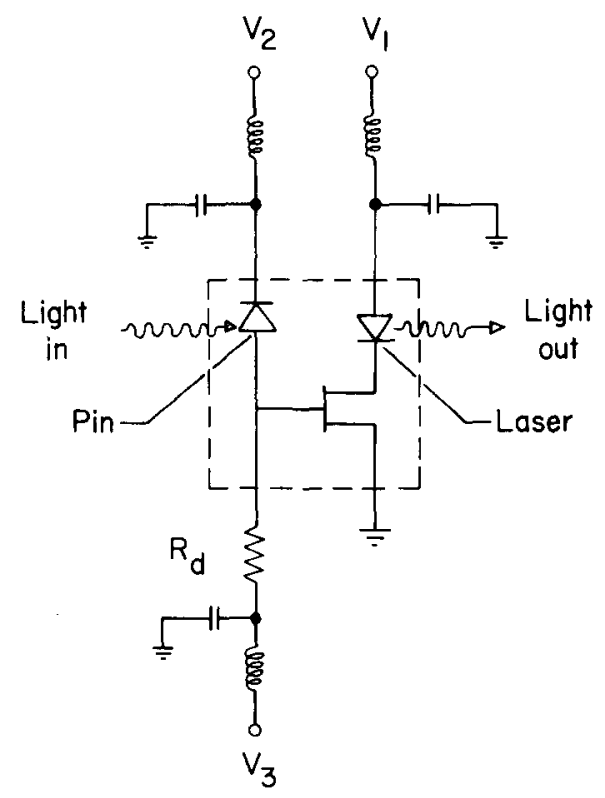

FIG. 1. Schematic diagram of the IOEC unit. Also shown are the bias networks for dc and rf testing of the circuit. flows through the laser is the product of the transconductance $\left(g_{m}\right)$ of the MESFET and the change in voltage which appears at the transistor gate. The dc optical gain of this repeater is

$$
G=\eta_{p d} \eta_{1} g_{m} R_{d}
$$

where $\eta_{p d}$ and $\eta_{l}$ are the quantum efficiencies of the photodiode and laser (from one facet), respectively. The $-3 \mathrm{~dB}$ bandwidth of the repeater is

$$
B=\frac{1}{2 \pi} \frac{1}{R_{d} C}
$$

where $C$ is the combined capacitance of the photodiode and MESFET. The gain bandwidth $(G \times B)$ product of the repeater is , therefore,

$$
G \times B=\eta_{\rho d} \eta_{l} g_{m} / 2 \pi C .
$$

A perspective view of the circuit is shown in Fig. 2. The fabrication steps which were used to realize the device are as follows. Epitaxial layers were grown on a semi-insulating GaAs substrate in the following order: $n$-GaAs layer $\left(n \simeq 2 \times 10^{16} \mathrm{~cm}^{-3}\right)$, lower cladding layer $\left(n-\mathrm{Ga}_{0.65} \mathrm{Al}_{0.35} \mathrm{As}\right)$, active layer (undoped $\mathrm{Ga}_{0.95} \mathrm{Al}_{0.05} \mathrm{As}$ ), and an upper cladding layer $\left(p-\mathrm{Ga}_{0.65} \mathrm{Al}_{0.35} \mathrm{As}\right)$. The $n$-GaAs layer performs three functions: it serves as the lower contact layer of the laser, the channel layer of the MESFET, and the photosensitive/contact layer of the photodiode. After epitaxial growth, mesas were etched down to the $n$-GaAs layer to form the

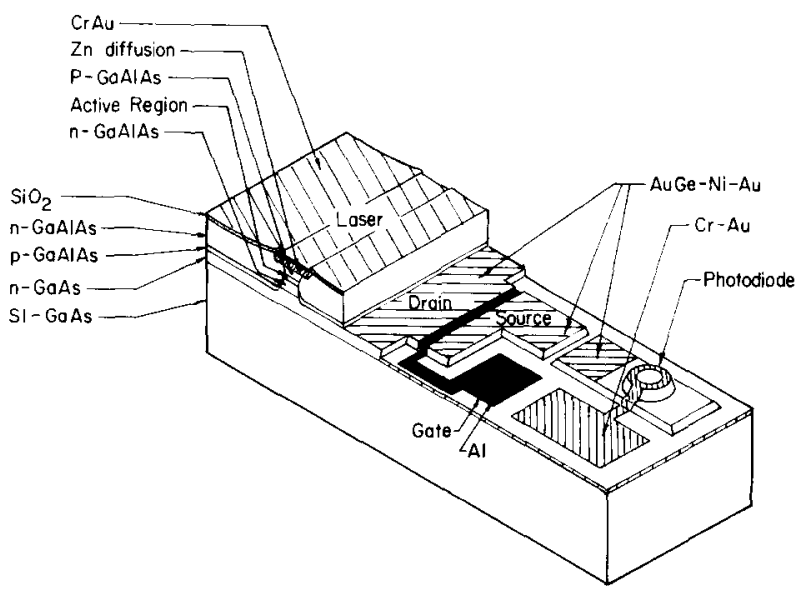

FIG. 2. Perspective view of the IOEC unit. 


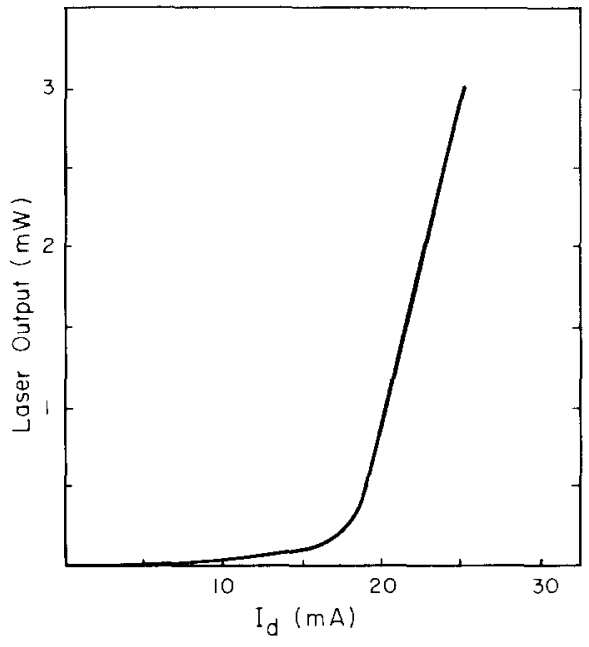

FIG. 3. Light vs current characteristics of the laser in the IOEC unit.

laser waveguides. The width of the active layer thus formed was $\simeq 2 \mu \mathrm{m}$.

Subsequent to the mesa etch, a $p-\mathrm{Ga}_{0.8} \mathrm{Al}_{0.2}$ As and an undoped $\mathrm{Ga}_{0.45} \mathrm{Al}_{0.55}$ As layer were regrown to embed the laser mesas. The $p-\mathrm{Ga}_{0.8} \mathrm{Al}_{0.2}$ As layer performs two functions: it serves as the leakage-current blocking layer of the laser and as the $p$-type window layer of the photodiode. The undoped $\mathrm{Ga}_{0.45} \mathrm{Al}_{0.55}$ As layer was then selectively etched with $\mathrm{HCl}$ everywhere except in the vicinity of the laser mesa. The exposed $p-\mathrm{Ga}_{0.8} \mathrm{Al}_{0.2}$ As layer was then etched to define the photodiodes. Mesa etching down to the substrate was used to isolate the devices from each other.

The entire wafer was next coated with $\mathrm{SiO}_{2}$. Windows were etched in the $\mathrm{SiO}_{2}$ to allow contact to be made to the $p$ type layers of the laser and the photodiode. $\mathrm{Zn}$ diffusion was performed to enhance the contact to the GaAlAs. $\mathrm{Cr}-\mathrm{Au}$ was deposited and etched to form contacts to the anodes of the laser and the photodiode. Contacts to the cathodes of the laser, photodiode, and the MESFET source and drain, were formed by etching through the $\mathrm{SiO}_{2}$, lifting off $\mathrm{AuGe} / \mathrm{Au}$, and alloying. The MESFET gates were formed by etching a recess into the $n$-GaAs MESFET channel until the desired thickness was obtained, and lifting off Al. The MESFET

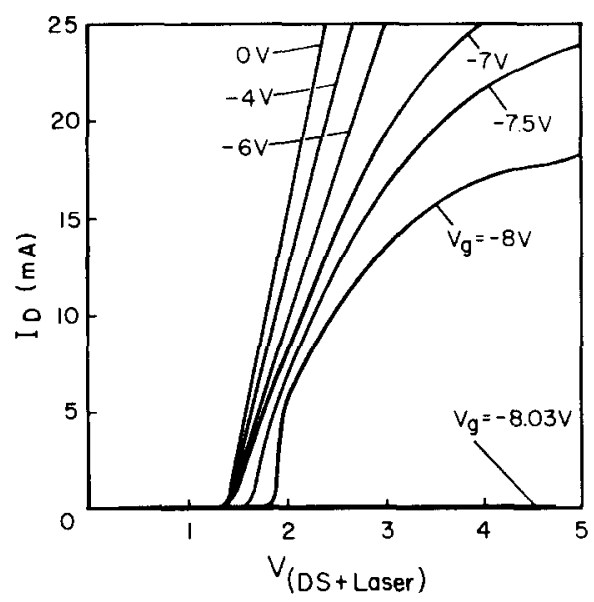

FIG. 4. Transconductance characteristics of the MESFET at various gate voltage. Note the sudden total pinchoff at $V_{g}=-8.03 \mathrm{~V}$. The channel does not recover until $V_{g}$ is increased to above $-7.7 \mathrm{~V}$.

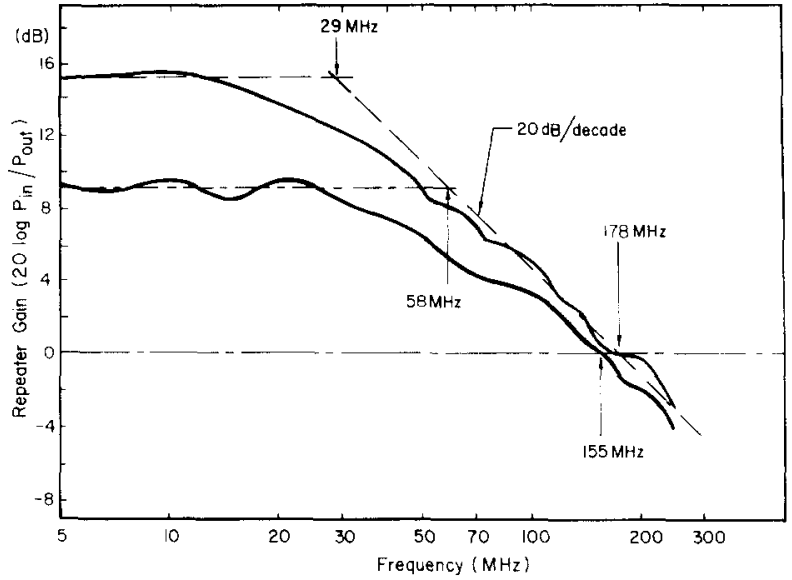

FIG. 5. Frequency response of the repeater unit at two values of the bias resistor $R_{d}$. Top curve $R_{d}=5.4 \mathrm{k} \Omega$, lower curve $R_{d}=2.7 \mathrm{k} \Omega$.

thus formed had a gate length of $2 \mu \mathrm{m}$ and a gate width of $250 \mu \mathrm{m}$.

The light vs current characteristic of the laser is shown in Fig. 3. The threshold current of 15-20 mA is typical of this laser structure. The transconductance characteristics of the FET are shown in Fig. 4, where the drain current is plotted against the voltage across the series combination of the laser and the FET, at various gate voltages. One interesting observation is that as the gate voltage is decreased from 0 to -8 $\mathrm{V}$, the drain current decreases continuously, but with a slight further decrease in the gate voltage (to $-8.03 \mathrm{~V}$ ) the FET is completely cut off and no current passes. The FET remains in the cut-off state unless the gate voltage is raised above $-7.7 \mathrm{~V}$, at which point the transistor resumes its normal transconductance characteristic. It is believed that this bistability is due to the coupling of the laser emission into the gate channel of the FET, which is sensitive to optical radiation. An increase in the gate voltage increases the drain current, and, hence, the amount of laser emission, which decreases the resistance of the gate channel by phototransistor or photoconductive effect ${ }^{2,3}$ and consequently further increases the drain current. This positive feedback effect can lead to a bistable characteristic as that observed in our devices. This bistable switching characteristic could be useful in digital repeater operation, since a small change in the gate voltage (caused by the incidence of a small optical pulse on the $p-i-n$ photodiode) can trigger a large change in the laser current. Unfortunately, this mode of operation cannot be demonstrated in our devices because the range of drain current where switching occurs is below the threshold current of the laser. In other words, the optical coupling between the laser and the FET channel in our devices is too strong. An optimum device structure design is required for the bistable mode of operation in this repeater unit.

The operation of our IOEC as a repeater was demonstrated using the circuit arrangement shown in Fig. 1. The load resistance $R_{d}$ shown in Fig. 1 was a chip resistor mounted next to the device. Laser emission from a separate GaAlAs laser diode is focused by a $20 \times$ objective on the $p-i$ $n$ detector in the IOEC unit. The absolute de responsivity of the $p-i-n$ diode was measured separately to be $0.45 \mathrm{~A} / \mathrm{W}$. The absolute amount of light falling on the $p-i-n$ detector 
can, thus, be determined by monitoring the dc photocurrent through $R_{d}$. The dc optical gain $G$ was measured to be $15 \mathrm{~dB}$ with $R_{d}=5.4 \mathrm{k} \Omega$, and $9.2 \mathrm{~dB}$ with $R_{d}=2.7 \mathrm{k} \Omega$. This is in good agreement with that expected by putting in the measured values of $\eta_{p d}(0.45 \mathrm{~A} / \mathrm{W}), G_{m}(6 \mathrm{mmho})$, and $\eta_{l}(0.4 \mathrm{~W} /$ A) into Eq. (1). The bandwidth of the repeater unit was measured by modulating the source laser and observing on a rf spectrum analyzer the modulation in the laser output from the IOEC unit with a separate $p-i-n$ detector. The combined frequency response of the external laser and detector was calibrated by repeating the measurement with the IOEC unit removed and the optical output from the external laser was directly detected by the external photodetector. The calibrated frequency responses of the repeater unit are shown in Fig. 5. The $-3 \mathrm{~dB}$ bandwidths are $29 \mathrm{MHz}$ with $R_{d}=5.4 \mathrm{k} \Omega$ and $52 \mathrm{MHz}$ with $R_{d}=2.7 \mathrm{k} \Omega$. The highfrequency falloff is at approximately $20 \mathrm{~dB} / \mathrm{dec}$, indicating that the response is manifested by a simple $R C$ combination. The observed bandwidth indicates a value of $C$ in the vicinity of $1 \mathrm{pF}$ according to Eq. (2). This value is consistent with that obtained by separate measurements ${ }^{4}$ and is within the range of values generally predicted for these kinds of devices. The measured gain bandwidth product was $178 \mathrm{MHz}$.

The above results illustrate clearly the operational principle of an optoelectronic repeater circuit. The simple singletransistor circuit described above has a $G \times B$ product of 178
MHz. It is obvious from Eq. (2) that $G \times B$ can be increased by improvement in the performance of the individual components in the IOEC unit (increasing $\eta_{p d}, \eta_{l}, g_{m}$, and decreasing $C$ ). However, it should be noted that the laser and detector in our repeater unit are already at the state-of-the-art level. Nonetheless, one can pick up a factor of 2 in $\eta_{l}$ just by reflection coating one face of the laser facet. The transconductance of the FET can be improved three to fourfold without introducing excessive gate capacitance by reducing the gate length to submicron dimensions. With these improvements, the $G \times B$ product of our device would exceed $1 \mathrm{GHz}$. This is the limit to the performance of a basic single-transistor repeater circuit. Achieving higher gain bandwidth products would require more complicated IOEC circuits containing multiple stage transistor amplifiers.

This research was supported by the Defense Advanced Research Project Agency and the Naval Research Laboratory.

\footnotetext{
'N. Bar-Chaim, I. Ury, and A. Yariv, IEEE spectrum 19, 38 (1982), and references contained therein.

${ }^{2}$ J. C. Gammel and J. M. Ballantyne, Proc. IEDM 120 (1978).

${ }^{3}$ T. Sugeta and Y. Mizushima, Jpn. J. Appl. Phys. 19, L27 (1980).

${ }^{4}$ N. Bar-Chaim, K. Y. Lau, I. Ury, and A. Yariv, Appl. Phys. Lett. 43, 261 (1983).
}

\title{
Temperature dependence of threshold current of injection lasers for short pulse excitation
}

\author{
N. K. Dutta and N. A. Olsson \\ AT\&T Bell Laboratories, Murray Hill, New Jersey 07974 \\ J. P. Heritage and P. L. Liu \\ Central Services Organization of the Regional Bell Operating Companies, Holmdel, New Jersey 07733
}

(Received 14 November 1983; accepted for publication 16 February 1984)

\begin{abstract}
We report measurements of the temperature dependence of the threshold current of GaAs, 1.3$\mu \mathrm{m}$ InGaAsP, and $1.5-\mu \mathrm{m}$ InGaAsP double heterostructure lasers using short electrical pulses. $T_{0} \sim 200 \mathrm{~K}$ is observed for all the lasers. These high $T_{0}$ values show that the carrier density at threshold does not increase rapidly with increasing temperature in any of the lasers studied and thus the observed low cw $T_{0}$ of InGaAsP lasers is primarily due to a decrease of the carrier lifetime at threshold with increasing temperature.
\end{abstract}

PACS numbers: $42.55 . \mathrm{Px}, 73.40 . \mathrm{Lq}$

The observed temperature dependence of threshold current of double heterostructure $(\mathrm{DH})$ semiconductor injection lasers can be represented by the expression $I_{\mathrm{th}}(T) \sim \exp \left(T / T_{0}\right)$, where the parameter $T_{0}$ is a measure of the temperature sensitivity.' ${ }^{\prime}$ The quantity $T_{0} \sim 180 \mathrm{~K}$ for GaAs-AlGaAs DH lasers and $T_{0} \sim 60 \mathrm{~K}$ for $1.3-\mu \mathrm{m}$ and 1.5 $\mu \mathrm{m}$ InGaAsP-InP DH lasers. ${ }^{1}$ The mechanisms proposed to explain the low $T_{0}$ of InGaAsP lasers are Auger recombination, ${ }^{2}$ carrier leakage over the heterobarrier, ${ }^{3}$ and inter-valence-band absorption. ${ }^{4}$
Recently $T_{0}$ values as high as $121 \mathrm{~K}$ have been observed using optical excitation with short pulses ${ }^{5}(\sim 100 \mathrm{ps})$ and $T_{0} \sim 148 \mathrm{~K}$ has been observed for a $1.3-\mu \mathrm{m} \mathrm{InGaAsP}$ laser using picosecond electrical pumping ${ }^{6}$ over a small temperature range $\left(12-25^{\circ} \mathrm{C}\right)$. This letter reports measured $T_{0}$ values $\sim 280 \mathrm{~K}$ for GaAs DH lasers, $\sim 222 \mathrm{~K}$ for $1.3-\mu \mathrm{m} \mathrm{InGaAsP}$ $\mathrm{DH}$ laser and $\sim 173 \mathrm{~K}$ for $1.5-\mu \mathrm{m} \mathrm{InGaAsP} \mathrm{DH}$ laser using short pulse current injection in the temperature range 10 $70^{\circ} \mathrm{C}$. These high $T_{0}$ values show that the carrier density at threshold does not increase rapidly with increasing tempera- 